\title{
Picking First Break in Seismic Traces by Fractal Method
}

\author{
Chang $\mathrm{Xu}^{*}$, Liu Yike* and Ashida Yuzuru** \\ * Institute of Geophysics, Chinese Academy of sciences \\ 11A Datun Road, Chaoyang District, Beijing, 100101 China \\ Email: changxu@mail.c-geos.ac.cn, ykliu@mail.c-geos.ac.cn \\ ** Department of Earth Resources Engineering, Faculty of Engineerung, Kyoto University \\ Yoshida-Honmachi, Sakyo-ku, Kyoto 606-8501, Japan \\ Email: ashida@tansa1.kumst.kyoto-u.ac.jp
}

\begin{abstract}
The determination of the first arrival time of seismic wave is an important factor in seismic tomography. The Hausdorff fractal dimension of seismic traces based upon fractal theory is different before and after first break. The sudden change point of fractal dimension for seismic traces indicates the position of the first arrival time. In the present paper, the method for calculating Hausdorff fractal dimension of seismic traces and its application to the detection of arrival time of the first break are proposed.
\end{abstract}

Key Word: Fractals, Fractal dimension, First break.

\section{Introduction}

The accurate determination of seismic traveltime is an important condition for evaluating the seismic velocities by using tomography. There are large velocity differences between the weathering layers in shallow subsurface and the deeper consolidated layers. It is very important to identify first arrival times for calculating the thickness and the velocity in shallow subsurface. Due to the complicated geological structure in shallow subsurface, the evaluation of the velocities of shallow subsurface with high accuracy is required.

Methods for automatically detecting the first break have been improved incessantly. In the recent years, many methods have been reported for automatically detecting first arrival times. For example, Gelchinsky and Shtivelman (1983) proposed a cross correlation method among adjacent traces. Coppens (1985) proposed a method for comparing the energy within different time windows in seismic trace. Ramananantoandro and Bernitsas (1987) proposed a convolution method. Boschetti (1996) proposed a method based on fractal dimensions. He showed that divider method and Hurst method can detect first arrivals of seismic waves. The key factors for most of automatic detection methods are based on the differences of seismic amplitude and frequency between first arrival point and its adjacent points in seismic trace.

In the present paper, we propose a method for automatic picking seismic first break by calculating Hausdorff fractal dimension along seismic traces. The seismic traces is composed of random noise before the first break, after the first break it is composed of seismic signal and noise. A change in Hausdorff fractal dimension is always found to indicate the transition from noise to 
signal plus noise. It is showed that this method is suitable for detecting first break from seismic data.

\section{The calculation of Hausdorff fractal dimension}

On the basis of fractals, space dimension is not only a discrete variable, but also, a continuous variable, which is either an integer or fraction. Any kinds of figures in Euclidean space can be expressed by equation (1) because fractal is not related to scale.

$$
A \propto r^{D}
$$

Where $A$ is a variable to describe an object, which may be length, area or volume, $r$ is a measured "ruler", and power exponent $D$ is Hausdorff fractal dimension. If an irregular curve length is measured by using a line segment with length $r$, its approximate length is $n r$, where $n$ is an integer number. Therefore, equation (1) is rewritten by equation (2).

$$
\text { nrocr } D
$$

According to the theory of self-similar in fractals, waveform curve in seismic traces (a time series) can be regraded as a statistical self-similar(Tucotte, 1992, Walden and Hosken, 1985). A number of different methods to calculate fractal dimension of self-similar curve or a seismic trace have been proposed(Mandelbrot, 1982). In the present paper, we use the method shown in Fig. 1 to calculate fractal dimension for seismic trace. The seismic trace is overlapped by a small circle moving along the seismic trace. When the whole curve is overlapped by the number of $n$ small circles, the length of curve is $L=n r$. Hausdorff fractal dimension of the curve can be obtained by equation (3)(kennedy and Lin, 1986) based on the fractal theory.

$$
D=1-\log (L) / \log (r)
$$

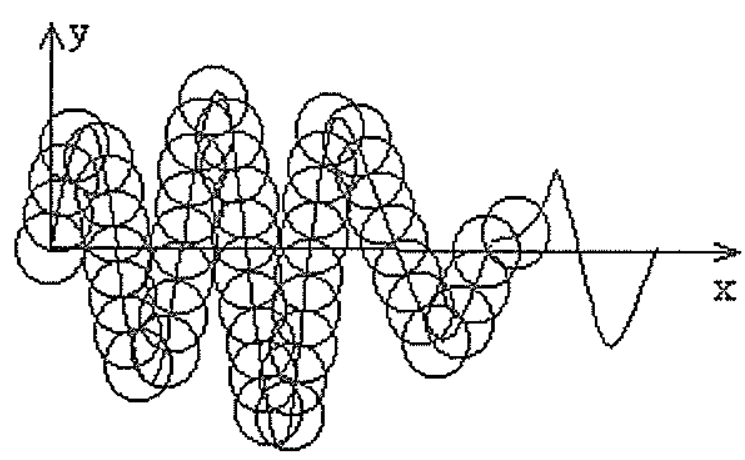

Fig. 1 Calculation method of fractal dimension of seismic trace.

In order to determine the arrival time of first break, we need to calculate the fractal dimension 
of every sample points along the curve. That is, the curve is dividered into many segments called as working windows along the horizontal axis. The working window is moved from first sample point to last sample point in seismic trace with one sample interval increment along the curve. In a working window, a number of the circles with different radius $r$ is used to calculate the curve length $L$. Different curve length $L$ is obtained by using different radius $r$. The smaller is the radius of circle, the longer is the calculated curve length, and the higher is the precision of the calculated curve length. Each of small circle is used to calculate one value of the curve length. There are the number of $i$ pairs of $r$ and $L$ if we use the number of $i$ small circles to measure the seismic trace length. All of the points $\left(\log \left(r_{i}\right), \log \left(L_{i}\right)\right)$ can be fitted as a linear function. According to fractal theory, this linear relation is called Mandelbrot-Richardson plot. In the study, Mandelbrot-Richardson plot is calculated in the every working window, and the fractal dimension of a working woindow is defined at last point of working window. Based on the Least Square method, Mandelbrot-Richardson plot is obtained from equation (4) in the $x-y$ coordinate system.

$$
Y(x)=a(x)+b
$$

Where, $a$ and $b$ are the parameters of linear equation and can be solved by equation (5).

$$
\left\{\begin{array}{l}
a \sum_{i=1}^{n} x_{i}^{2}+b \sum_{i=1}^{n} x_{i}=\sum_{i=1}^{n} x_{i} y_{i} \\
a \sum_{i=1}^{n} x_{i}+b n=\sum_{i=1}^{n} y_{i}
\end{array}\right.
$$

Here, $x_{i}$ and $y_{i}$ indicate $\log \left(r_{i}\right)$ and $\log \left(L_{i}\right)$, respectivly. In equation (5), $i$ means the number of small circles with different diameters in working window. Therefore, the fitting curve determined by $\log \left(r_{i}\right)$ and $\log \left(L_{i}\right)$ is obtained by putting $a$ and $b$ values into equation(4). The slope $a$ of Mandelbrot-Richardson plot is a basic data for calculating the fractal dimension, which is obtained by equation (6).

$$
a=y_{i} / x_{i}=\log \left(L_{i}\right) / \log \left(r_{i}\right)
$$

According to equation (3) and equation (6), Hausdorff fractal dimension of seismic trace is calculated by equation (7).

$$
D=1-a
$$

Fig. 2 (a) shows a seismic trace. Fig. 2 (b) shows a Mandelbrot-Richardson plot in a working window in the seismic trace shown in fig. 2 (a).

According to fractal dimension method mentioned above, we can acquire the fractal dimensions at every point of the curve. For the seismic traces, the values of amplitudes include 
only random noise before the first break, and after the first break, the values of amplitudes include the noise and seismic wave. It can be known that the fractal dimension of seismic trace is different before and after the first break. This means that fractal dimension describes quantitatively the figure shape of seismic traces. The change of fractal dimension corresponds to the change of waveform in seismic trace. The abrupt change of fractal dimension corresponds to the abrupt change of waveform. Therefore, the fractal dimension of seismic trace indicates the position of first break. The reasonableness for calculation of fractal dimension of seismic trace is influenced by following two points.

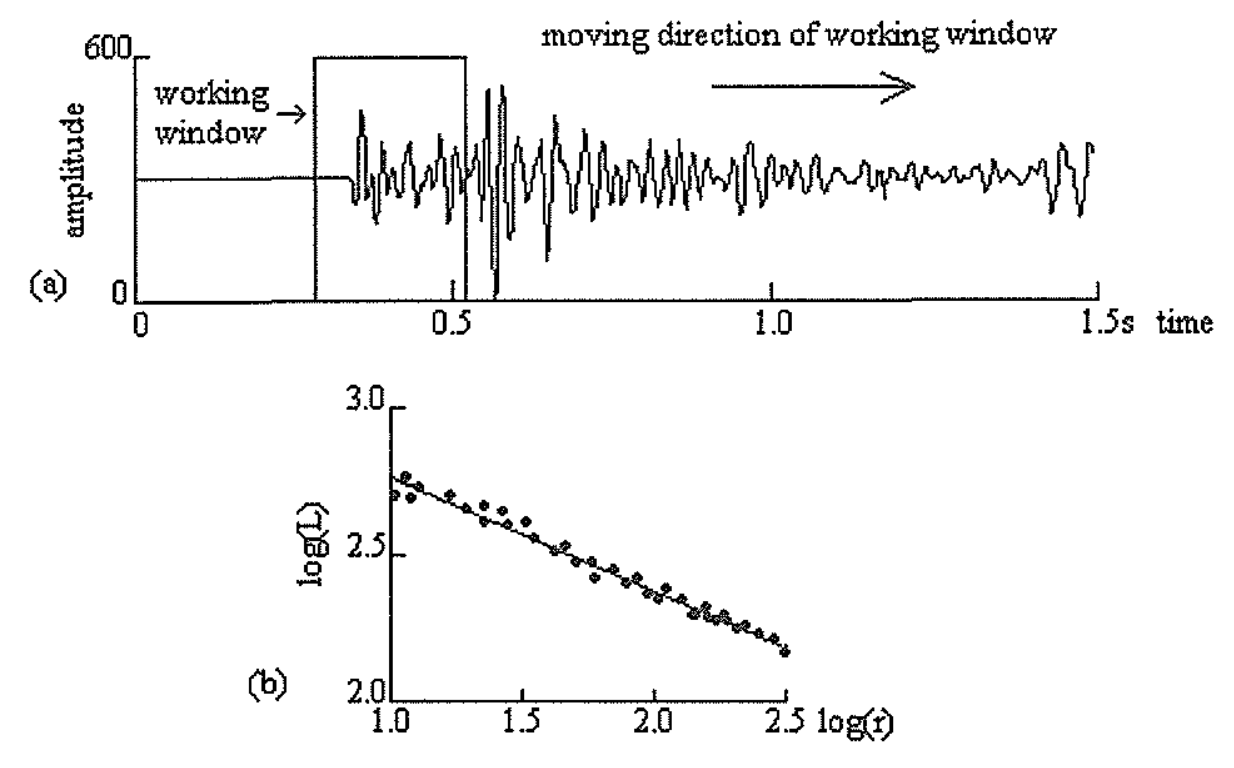

Fig. 2 (a), seismic trace. (b), Mandbrot-Richardson plot for a working window in the seismic trace.

The first is the concordant of the curve shape in the $x-y$ coordinate system( $x$ direction means traveltime and $y$ direction means amplitude). If the value in $y$ - direction is much bigger than the samples interval in x-direction, the accumulative calculation error is easy occured in the computation. Therefore, in our study, the curve of seismic trace was reformed to a concordant shape by changing the both scales of time and amplitude coordinate axis.

The second is to select the diameter of the small circles. The minmum radius of the small circle should be 2 - 4 sample interval in order to reduce unnecessary amount of calculation. The maximum radius should be smaller than half length of working window. It is easy to understand from Fig. 3, if the maximum radius of the small circle is bigger than the length of working window or the maximum amplitude of the seismic trace within the working window, $n$ is zero, and also the calculated length of the curve $L=n r$ will be zero. This is beyond the definition region of $\log (L)$. The optimum increment of the diameter is related to the number of points in working 
window.

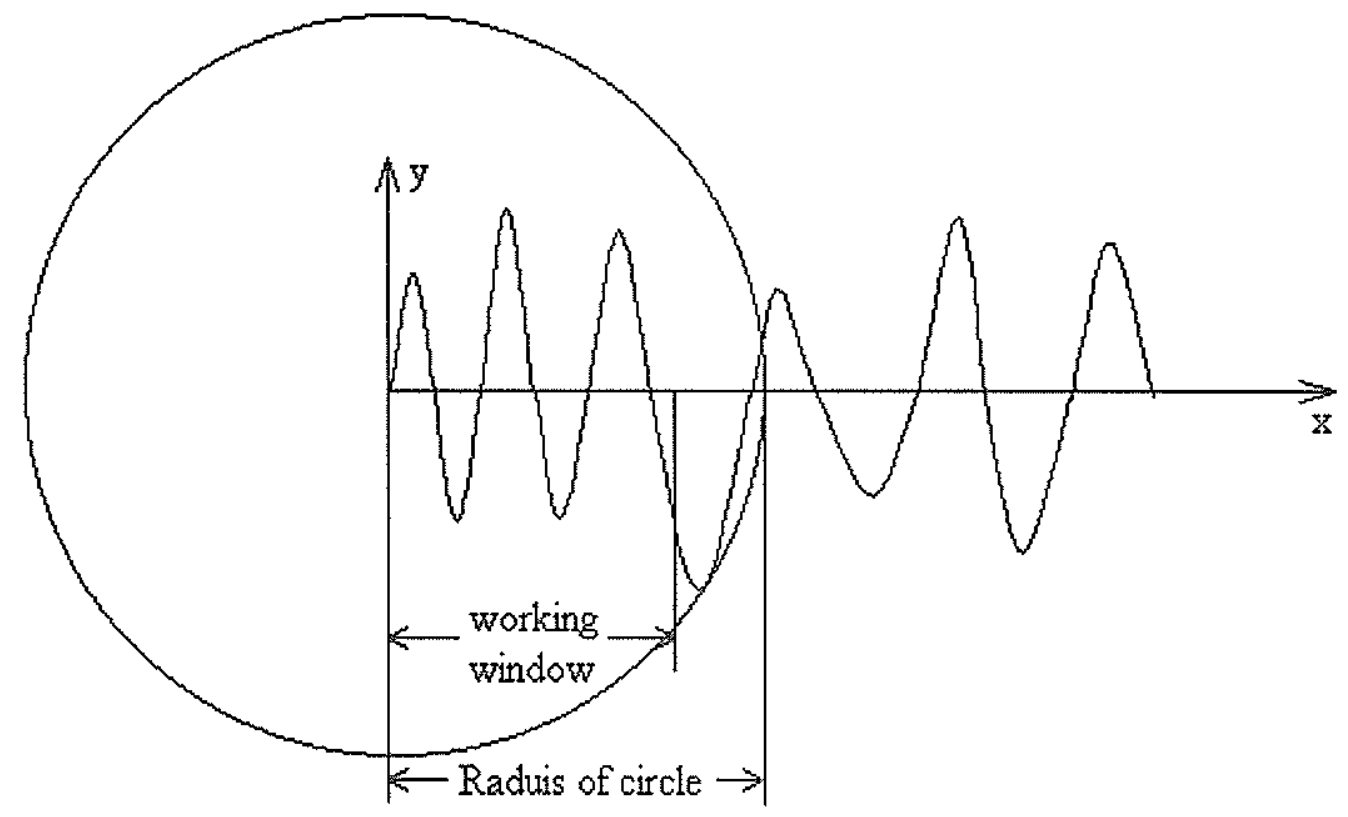

Fig. 3 Relationship between radius of circle and working window lenght. If the radius of the circle is bigger than the lenght of the working window, the calculated lenght of curve is almost equal to zero.

\section{Application to detection of first break in seismic traces}

In our study, in order to calculate the fractal dimension of seismic trace, the minimum radius, the maximum radius and the increment of the radius is defined as two sample intervals, fifty sample intervals and one sample interval along the horizontal axis, respectively. The working window is selected to one hundred smple intervals. The relationship between minimum radius, maximum radius and calculated length of the curve in a working window are shown in Fig. 4.

Fig. 5 shows the calculation results by use of the field observed data for high resolution seismic tomography. The Hausdorff dimension does not change until the first break appears, and the abrupt change point of Hausdorff dimension indicates clearly the position of arrival time of first break.

\section{Summary}

The present study illustrates that Hausdorff fractal dimension can be used to detect automaticly and quantitatively the first break of seismic trace. The calculation results stated that there is difference of fractal dimension of seismic wave with time change, and the abrupt change point of 
fractal dimension corresponds the first break. The calculation results show special advantages of this method. That are, (1) the calculation of fractal dimension is only related to single trace, which means that it is not restrained by the adjacent traces and observation systems, (2)quantitative degree in this method is higher. These advantages show a good practical application prospects.
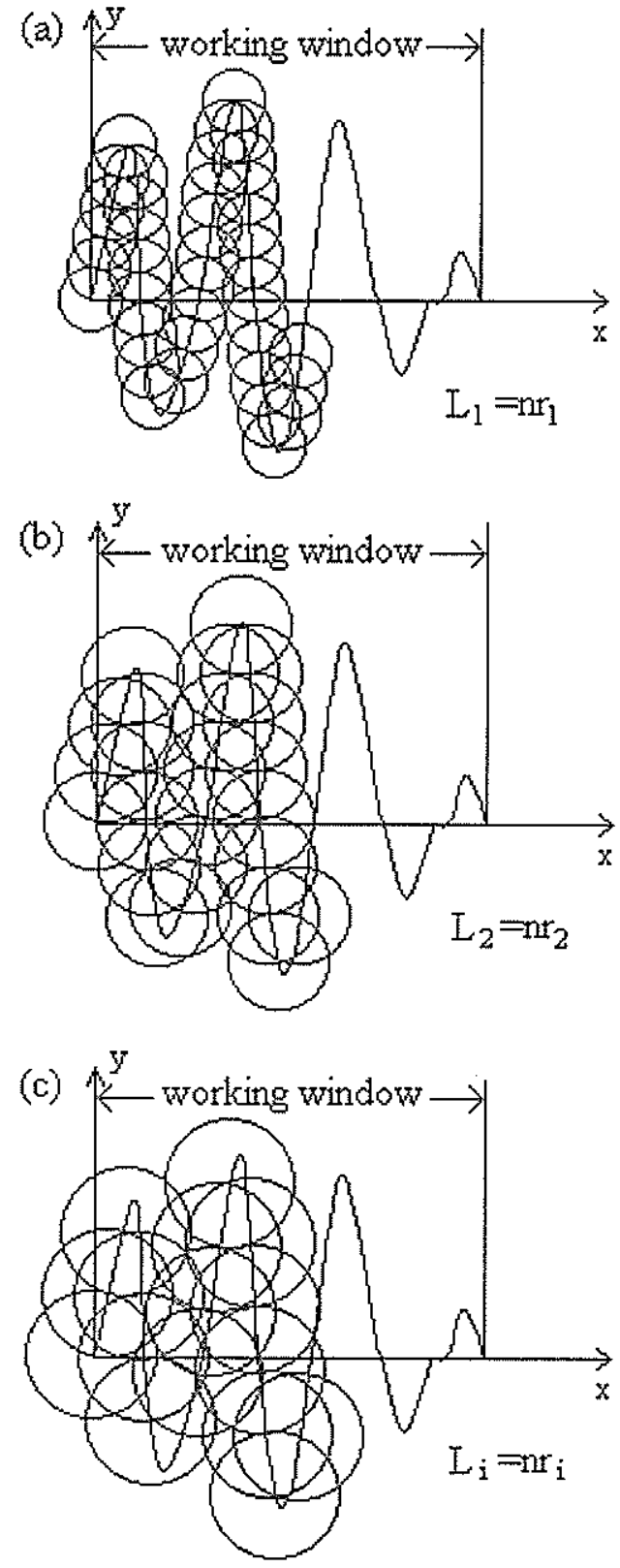

Fig. 4 The different calculated length of curve is obtained by using different small circle with vary daimeters. 
It should be noted that if the signal to noise ratio is very low, so as to there is not the difference in the figure shape of seismic amplitude with time change before and after the first break, the fractal dimension of the amplitudes do not have the characteristic stated in the present paper. However, the frequency difference between noise and signal reminds us that we can calculate the fractal dimensions when the noise is suppressed by filter.

The method proposed in the present paper is suitable for the automatic detection of traveltime to various regular and irregular seismic servey and natural earthquake. It can be used to detect the first break of seismic trace.

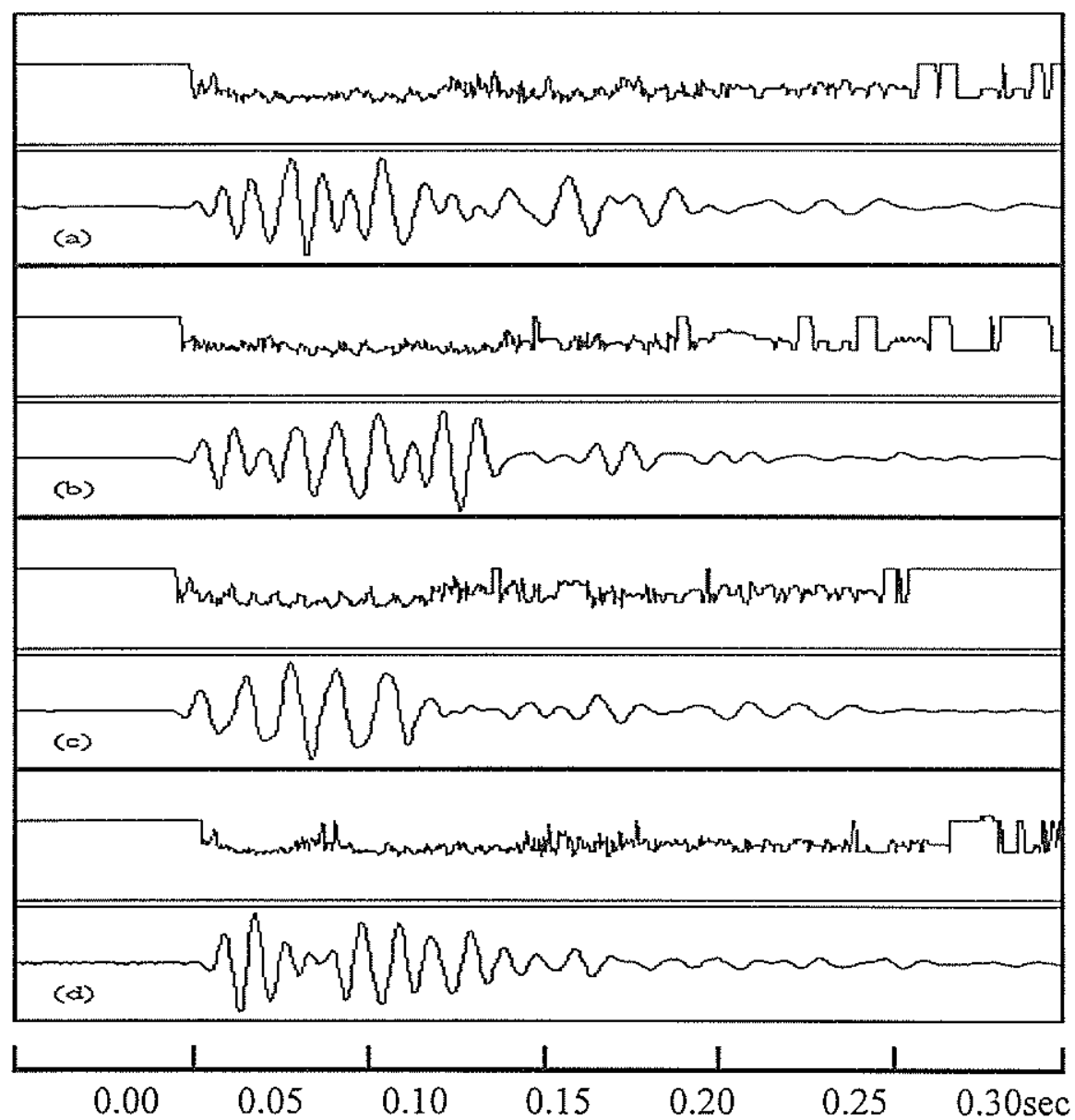

Fig. 5 a, b, c and d shows a pair of seismic trace(below) and its fractal dimension curve(above), respectively. Vertical axis at below means the the amplitude with value from -1 to 1 . Vertical axis at above means the fractal dimension with value from 1.0 to 1.4 . 


\section{Acknowledgments}

This study was supported by Chinese National Natural Sciences Foundation(49674236). We thank Toshifumi Matsuoka of Kyoto Univervsity for giving us a lot of suggestion in our study.

\section{References}

Boschetti F., Dentith D. M. and List D. R., Afractal-based algorithm for detecting first arrivals on seismic traces, Geophysics, Vol. 61, p. 1095-1102, 1996.

Coppens F., First arrival picking on common-offset trace collections for automatic estimation of static corrections, Geophys. Prosp., Vol. 33, p. 1212-1223, 1985.

Geolchinsky B. and Shtivelman V., Automatic picking of first arrivls and parameterization of traveltime curves, Geophys. Prosp., Vol. 31, p. 915-928, 1983.

Kennedy S. K. and Lin W., FRACT-A fortran subroutine to calculate the variables necessary to determine the fractal dimension of closed forms, Comput. and Geosci., Vol. 12, 705-712, 1986.

Ramananantoandro R. and Bernitsas N., A commputer algorithm for automatic picking of refraction first-arrival time, geoexploration, Vol. 24, p. 147-151, 1987.

Tucotte D., Fractals and Chaos in geology and geophysics, Canbridge Univ. Press, 1992.

Walden A. T. and Hosken J. W., An investigation of the spectral properties of primary reflection coefficients, Geophys. Prosp., Vol. 33, p.400-435, 1985.

Mandelbrot B. B., The fractal geometry of nature, Freeman, San Francisco, 1982. 